\title{
HEISENBERG ANTIFERROMAGNET ON A 1/7-DEPLETED TRIANGULAR LATTICE
}

\author{
J. Schulenburg ${ }^{a}$, J. Richter ${ }^{a}$ And D.D. BetTs ${ }^{b}$ \\ ${ }^{a}$ Institut für Theoretische Physik, Uni Magdeburg, Germany \\ ${ }^{b}$ Department of Physics, Dalhousie University, Halifax, NS, B3H 3J5, Canada
}

\begin{abstract}
Based on the triangular lattice and its depletions there are three simple frustrated antiferromagnetic Heisenberg models in two dimensions. The first two, the triangular and kagomé lattices, have been examined in the recent past. The triangular lattice seems to have a long range order whereas the kagomé does not show the long range order. But these results are still controversial. This work is concentrated on a third type of this lattice family in order to improve the understanding of the connection between the long range order and coordination number in low dimensional systems. Bets has described the geometric properties of this lattice. It has a coordination number 5 , which lies precisely between coordination numbers 6 and 4 of the other two lattices. The low-lying spectra and the correlation functions of finite lattices have been examined to discuss the possibility of a long range ordered ground state in the 1/7-depleted triangular lattice. The low-lying spectrum is generated by an exact diagonalization, and the tower of states behavior points to a long range ordered ground state.

PACS numbers: $75.10 . \mathrm{Hk}, 75.10 . \mathrm{Jm}, 75.40 . \mathrm{Mg}, 75.50 . \mathrm{Ee}$
\end{abstract}

\section{Introduction}

The presence of a long range ordered (LRO) ground state in two-dimensional spin-1/2 Heisenberg antiferromagnets is still an open question. Much attention has been paid to frustrated systems since frustration is one mechanism to suppress the ground state Néel order. One example is the frustrated antiferromagnet (AFM) on the triangular lattice as a Bravais lattice. The study of the triangular spin$-1 / 2$ Heisenberg AFM goes back to early papers of Anderson [1,2] who argued that the ground state is a spin liquid. However, as a result of intensive studies of this problem in the nineties (see e.g. [3-5]) it seems to be consensus that the ground state of the triangular Heisenberg AFM is Néel-like ordered. That may be related to the large number of neighbors per site. A candidate for spin liquid behavior should have a small coordination number $z$ and frustration. The quantum Heisenberg AFM on the kagome lattice has exactly these features and recent investigations $[6,7]$ support the existence of a disordered ground state.

The aim of this paper is to study the spin- $1 / 2$ Heisenberg AFM on a regular two-dimensional lattice with a coordination number $z=5$, halfway between the coordination numbers of the kagomé and triangular lattices. 


\section{Depletions of the triangular lattice}

It was shown in [8] that the regular depletion of the triangular lattice by $1 / 7$ yields exactly a lattice with a coordination number $z=5$. Like the kagomé lattice the 1/7-depleted lattice is a non-Bravais lattice with a hexagonal unitcell. But the number of sites in one unitcell is six. The 1/7-depleted lattice has a 6 -fold rotational symmetry but no reflection symmetry. The kagomé lattice and the honeycomb lattice can also be derived from the triangular lattice by depletion of $1 / 4$ or $1 / 3$, respectively. The AFM on the honeycomb lattice is not frustrated and we will not discuss it here. The 1/4- and 1/7-depleted lattices are two-dimensional regular lattices containing only triangles and hexagons. According to the recent conjecture given in [9] these lattices belong to the class of even-odd lattices and are therefore candidates for the systems having a non-Néel-like quantum ground state.

\section{Classical ground state}

The ground state of the classical AFM on the triangular lattice is a three-sublattice Néel state with an angle of $2 \pi / 3$ between spins of different sublattices. There is no nontrivial degeneracy on the triangular lattice. For the kagomé lattice there is a large number of nontrivial degenerated ground states which can be divided into two main classes $[10,11]$. The first type is the $q=0$ configuration. The second one is the $\sqrt{3} \times \sqrt{3}$ configuration. The corresponding planar states where neighboring spins are rotated by $2 \pi / 3$ also belong to both configurations.

The ground state on the 1/7-depleted lattice is planar and has three different angles between neighboring spins. The angle between neighboring spins on a hexagon is $5 \pi / 6$. On each elementary triangle connected to three hexagons the classical spins in the ground state make $2 \pi / 3$ angles with one another. On the one remaining bond the angle between spins is $\pi / 2$ (see Fig. 1). Obviously, this ground state has some relation to the three-sublattice Néel state of the triangular lattice:

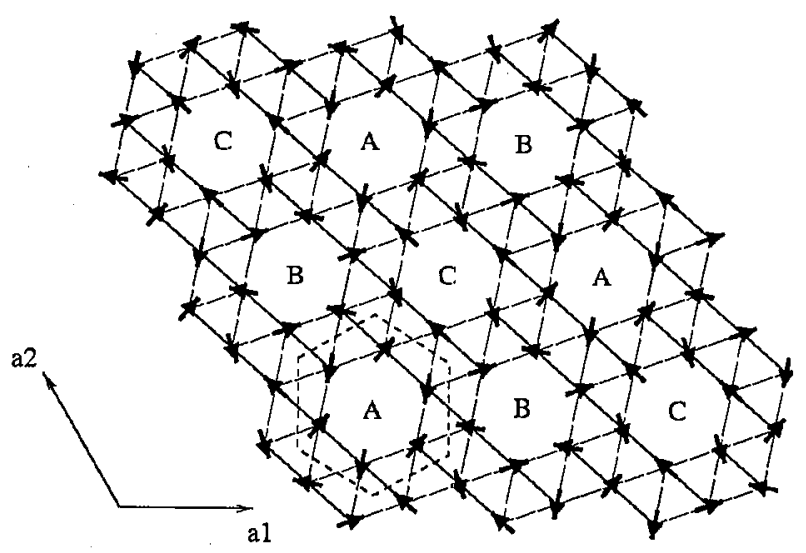

Fig. 1. The 1/7-depleted lattice and the ground state for a classical antiferromagnet with the same structure. The dashed hexagon shows the Wigner-Seitz cell of the lattice. 
considering hexagons we have three sublattices of hexagons $A, B, C$ with the angle $2 \pi / 3$ between corresponding spins of nonequivalent hexagons. The energy of this ground state is $-(\sqrt{3}+1) / 5$ per bond whereas the energy per bond for the triangular lattice and the kagomé lattice is -0.5 . Since the classical ground state energy can be considered as a measure of frustration strength we argue that all three lattices are almost equally frustrated.

\section{4. $S=1 / 2$ quantum case}

We use an exact diagonalization of small lattices to get the ground state and the low-lying levels for the 1/7-depleted lattice. This method was successfully applied also to the triangular and kagomé lattices $[3,6,7]$. Using $S^{z}$ and lattice symmetries we are able to diagonalize finite systems up to $N=24$ and partly $N=30$. Because of the lower symmetry compared with the triangular and kagomé lattices the diagonalization of $N=36$ is much more difficult.

Only lattices with $N$ being a multiple of $18(N=18,36)$ fit to the classical ground state configuration of the infinite lattice. Hence $N=24$ and $N=30$ are less appropriate but they should be considered nevertheless. Though the lattice is non-bipartite, similarly to the triangular and kagomé lattices we find a singlet ground state and a level ordering with increasing $S$ which fits to the Lieb-Mattis theorem [12] for bipartite lattices. Figure 2 shows the spectra of the $N=18$ and $N=24$ lattices. The "tower of states" behavior $E=S(S+1) / I$ can be clearly seen in both diagrams. The slope of this line goes rapidly to zero $I \propto N$. This is a necessary condition to get a symmetry breaking ordered state for the thermodynamical limit. Similarly to the triangular lattice and in contrast to the

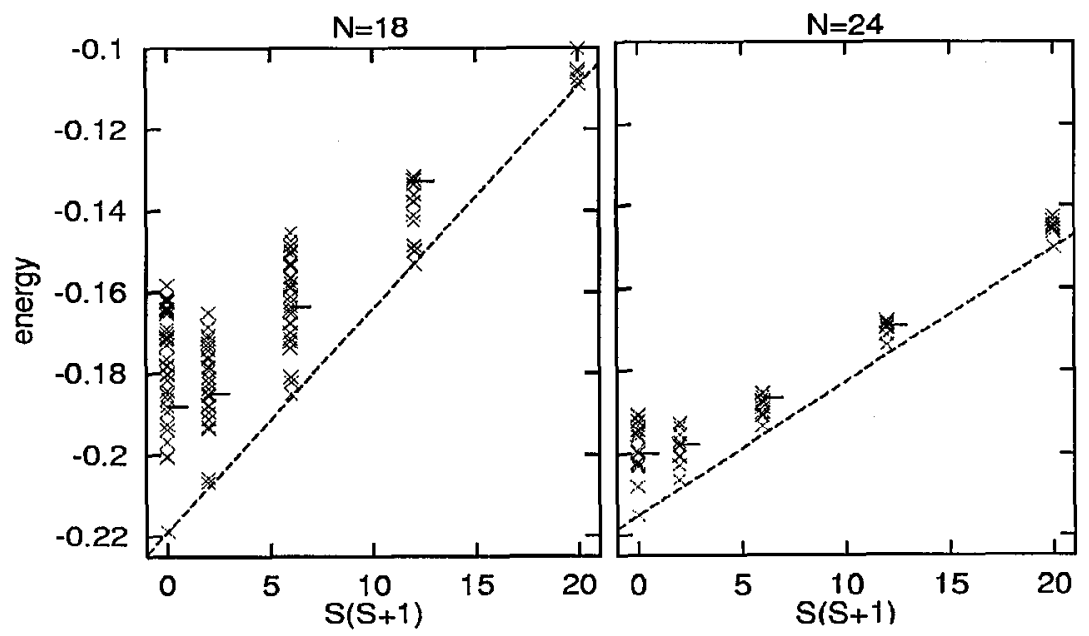

Fig. 2. The low-lying spectrum of the 1/7-depleted lattice for finite cells with $N=18$ and $N^{\circ}=24$ and periodic boundary conditions. The straight line is only a guide for the eyes to show the linear dependence of the lowest energy in every $S$-subspace. The horizontal lines mark the highest energies where all eigenvalues have been computed. 


\section{TABLE}

Three types of spin-spin correlations between next-neighbors are presented. The largest correlation is placed around a hexagon, the lowest is placed on the dimer bond connecting two hexagons. The singlet-triplet gap $\Delta_{\mathrm{t}}$ and singlet-singlet gap $\Delta_{\mathrm{s}}$ are also shown.

\begin{tabular}{c|c|c|c|c|c}
\hline \hline$N$ & $\left\langle\boldsymbol{S}_{i} \boldsymbol{S}_{j}\right\rangle_{\text {hex }}$ & $\left\langle\boldsymbol{S}_{i} \boldsymbol{S}_{j}\right\rangle_{\text {tri }}$ & $\left\langle\boldsymbol{S}_{i} \boldsymbol{S}_{j}\right\rangle_{\mathrm{di}}$ & $\Delta_{\mathrm{t}}$ & $\Delta_{\mathrm{s}}$ \\
\hline 18 & -0.366673 & -0.186299 & 0.010923 & 0.012115 & 0.018330 \\
24 & -0.373706 & -0.160023 & -0.008695 & 0.008800 & 0.007131
\end{tabular}

kagomé lattice the lowest levels are lying well on a straight line and there are no or only a few states between the ground state and the lowest triplet excitation. The nearest-neighbor spin-spin correlations are shown in Table. Analogously to the classical case there are three different nearest-neighbor correlations corresponding well to the classical ground state; for example the strongest one is placed around the hexagons and the correlation $\left\langle\boldsymbol{S}_{i} \boldsymbol{S}_{j}\right\rangle_{\mathrm{di}}$ of classically perpendicular spins is very small.

\section{Conclusions}

In conclusion, we consider for the first time the Heisenberg AFM frustrated on a two-dimensional 1/7-depleted triangular lattice with a coordination number of five. We find that the Heisenberg AFM on this lattice is more similar to the Heisenberg AFM on the triangular parent lattice than to the Heisenberg AFM on the kagomé (i.e. the 1/4-depleted triangular) lattice. This conclusion is based on the analysis of (i) the classical ground state, (ii) the spin correlation, and (iii) the low-lying energy spectra of finite lattices. We argue that our results may indicate the possibility of a multi-sublattice Néel-like quantum ground state in a lattice built by odd and even polygons.

\section{References}

[1] P.W. Anderson, Mater. Res. Bull. 8, 153 (1973).

[2] P. Fazekas, P.W. Anderson, Philos. Mag. 30, 423 (1974).

[3] B. Bernu, P. Lecheminant, C. Lhuillier, L. Pierre, Phys. Rev. B 50, 10048 (1994).

[4] G. Misguich, C. Lhuillier, B. Bernu, C. Waldtmann, preprint available on http://xxx.lanl.gov/cond-mat/9812329.

[5] R. Deutscher, H.U. Everts, Z. Phys. B 93, 77 (1993).

[6] P. Lecheminant, B. Bernu, C. Lhuillier, L. Pierre, P. Sindzingre, Phys. Rev. B 56, 2521 (1997).

[7] Ch. Waldtmann, H.U. Everts, B. Bernu, C. Lhuillier, P. Sindzingre, P. Lecheminant, L. Pierre, Eur. Phys. J. B 2, 501 (1998).

[8] D.D. Betts, Proc. N. S. Inst. Sci 40, 95 (1995).

[9] P. Tomczak, J. Richter, Phys. Rev. B 59, 107 (1999).

[10] A. Chubukov, Phys. Rev. Lett. 69, 832 (1992).

[11] J.N. Reimers, A.J. Berlinsky, Phys. Rev. B 48, 9539 (1993).

[12] E. Lieb, D. Mattis, J. Math. Phys. 3, 749 (1962). 